\title{
Stimulus exposure and gaze bias: A further test of the gaze cascade model
}

\author{
Mackenzie G. Glaholt and Eyal M. Reingold \\ University of Toronto at Mississauga, Mississauga, Ontario, Canada
}

\begin{abstract}
We tested predictions derived from the gaze cascade model of preference decision making (Shimojo, Simion, Shimojo, \& Scheier, 2003; Simion \& Shimojo, 2006, 2007). In each trial, participants' eye movements were monitored while they performed an eight-alternative decision task in which four of the items in the array were preexposed prior to the trial. Replicating previous findings, we found a gaze bias toward the chosen item prior to the response. However, contrary to the prediction of the gaze cascade model, preexposure of stimuli decreased, rather than increased, the magnitude of the gaze bias in preference decisions. Furthermore, unlike the prediction of the model, preexposure did not affect the likelihood of an item being chosen, and the pattern of looking behavior in preference decisions and on a nonpreference control task was remarkably similar. Implications of the present findings in multistage models of decision making are discussed.
\end{abstract}

Shimojo, Simion, and colleagues (Shimojo, Simion, Shimojo, \& Scheier, 2003; Simion \& Shimojo, 2006, 2007) reported a bias in looking behavior during twoalternative forced choice (2-AFC) preference decisions. Shimojo et al. found that when participants chose the more attractive of two faces, their gaze became increasingly biased toward the chosen stimulus, prior to their response. To explain this effect, Shimojo et al. proposed a model, referred to as the gaze cascade model, in which two component processes related to looking behavior interact to produce the observed gaze bias in preference decisions. One process is the mere exposure effect, where looking at a stimulus increases preference for that stimulus (KunstWilson \& Zajonc, 1980; Moreland \& Zajonc, 1977, 1982; Zajonc, 1968). The other process is preferential looking, where one tends to look longer at the stimulus that one likes (Birch, Shimojo, \& Held, 1985; Fantz, 1964). Shimojo and colleagues suggested that these two processes can combine to create a positive feedback loop (i.e., a gaze cascade) that progressively increases the activation of one of the decision options until it exceeds the threshold for response (for the most recent description of this model, see Simion \& Shimojo, 2007, p. 591). Shimojo and colleagues acknowledged that the gaze cascade is by no means the only determinant of preference decisions and that other factors, such as preexisting preferences, influence the outcome of decision processes. However, all other factors being equal, the positive feedback loop postulated by the gaze cascade model (see Figure 1) is expected to produce a substantial influence on preference decisions.
Due to its reliance on preference-specific mechanisms, the gaze cascade model predicts that the gaze bias should be uniquely present in preference decisions. To test this prediction, Shimojo et al. (2003) contrasted preference decisions with a control task in which participants judged which of two faces was rounder (the roundness decision). The gaze bias was significantly more pronounced in preference decisions than in the roundness decision. In a second experiment, Shimojo et al. demonstrated that longer exposure to a face stimulus increases the likelihood of choosing that face in a 2-AFC preference decision, but not in a 2-AFC roundness decision.

To test the generality of the findings reported by Shimojo and colleagues, we introduced an eight-alternative forced choice (8-AFC) paradigm where participants made preference decisions among eight photographs (Glaholt \& Reingold, in press). A robust gaze bias was observed in the 8-AFC decisions. In addition, an analysis of the dwell sequence during the trial (a dwell being a series of one or more consecutive fixations on a single alternative) demonstrated a bias in both dwell duration on the chosen item and in dwell frequency on the chosen item. Specifically, from the very first dwell and thereafter throughout the trial, dwells on the chosen item were longer than were dwells on other items, and dwell frequency on the chosen item was significantly greater than chance in the last few dwells prior to response. However, in contrast to the prediction of the gaze cascade model, a comparison of a preference decision task and a control task in which participants decided which photograph was taken most recently (the recency decision) revealed a very similar

M. G. Glaholt, mackenzie.glaholt@gmail.com 


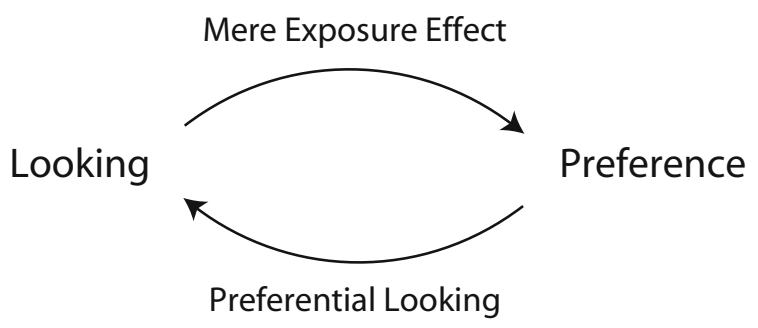

Figure 1. The positive feedback loop between preference and looking behavior described in the gaze cascade model (Simion \& Shimojo, 2007).

pattern of gaze bias across tasks. Accordingly, we argued that the observed gaze bias pattern might reflect a general phenomenon in visual decision making rather than being restricted to preference decisions, as suggested by the gaze cascade model.

The main goal of the present study was to provide a further test of the gaze cascade model by manipulating stimulus exposure. On each trial, differential exposure across alternatives was produced by providing a preview of four of the eight alternatives prior to the 8-AFC preference decision. The gaze cascade model predicts that differential stimulus exposure across alternatives would result in a stronger gaze bias effect for items that were preexposed which, in addition, would be predicted to have a greater likelihood of being chosen in the preference decision task (Shimojo et al., 2003; see Figure 1). To examine the task specificity of any observed effects of preexposure, we contrasted the preference decision task with a control task in which participants were asked to choose the most unusual photograph out of eight alternatives (the typicality decision).

\section{METHOD}

\section{Participants}

All participants were undergraduate students at the University of Toronto at Mississauga, and each received $\$ 10$ for participation. Decision was manipulated between participants, with 16 participants performing the preference decision and 16 participants performing the typicality decision.

\section{Apparatus}

The eyetracker employed in this research was an SR Research Ltd. EyeLink 1000 system. Following calibration, gaze-position error was less than $0.5^{\circ}$. Stimulus displays were presented on a 19-in. monitor. Each participant's monitor was set to a resolution of $1,600 \times 1,200$ pixels and a refresh rate of $85 \mathrm{~Hz}$. Participants were seated $60 \mathrm{~cm}$ from the display, and a chinrest with a head support was used to minimize head movement.

\section{Materials and Design}

Stimuli were drawn from a set of 384 grayscale images of photographic art. The photographs varied widely in style and subject matter: portraits, landscapes, social interactions, objects, architecture, and so on (for more details, see Glaholt \& Reingold, in press). These photographs were used to create 48 8-AFC experimental trials. Additional stimuli not used in experimental trials appeared in 4 practice trials, which served to familiarize the participant with the task.

Decision task was a between-participants variable. In the preference task, the participant was instructed to select from the eight alternatives the image that he/she liked the most. In the typicality task, the participant had to select the image that he/she judged to be most unusual (i.e., most out of the ordinary, least typical). Across images, there was no significant correlation between the number of times an image was selected as the preferred image and the number of times it was selected as the most unusual (Pearson's $r=.08$, n.s.). The eight stimuli for the 8-AFC decision in each trial were presented in a $3 \times 3$ array, where each cell measured $8^{\circ} \times 8^{\circ}$ of visual angle $(400 \times 400$ pixels). The middle cell was empty except for a fixation circle (see Figure 2).

\section{Procedure}

The trial sequence began with the presentation of four (randomly selected) of the eight stimuli for that trial. The four stimuli were centrally presented, one after the other, for $1 \mathrm{sec}$ each, with a $500-\mathrm{msec}$ blank interval between them. Following this preexposure phase, there was a 2-sec blank interval, after which all eight images were presented in a $3 \times 3$ grid (gridlines were shown as 1-pixel-wide black lines). Having reached a decision, the participant looked at a gray circle at the center of the screen and pressed a button on a button box. This caused the circle to turn green, which signaled the participant to then fixate the chosen item in order to select it. After the participant had gazed at his/her choice for $300 \mathrm{msec}$, a chime sounded and the trial ended. The participant then advanced to the next trial by fixating the central (empty) grid square and pressing a button on a button box.

\section{RESULTS}

As discussed earlier, a critical prediction of the gaze cascade model is that preexposed items would be more likely to be chosen in preference decisions. However, our data did not support this prediction, since the likelihood of choosing a preexposed item did not differ from chance in the preference task (prob. $=.52$; chance $=.5 ; t<1$ ). In addition, preexposure did not affect choice in the typicality task (prob. $=.48$; chance $=.5 ; t<1$ ).

Following Shimojo et al. (2003), we produced gaze likelihood analysis plots that displayed the proportion of time that a participant's gaze was directed at the chosen item just prior to the response. The analysis window spanned $2.5 \mathrm{sec}$. The gaze likelihood plots for each decision type, averaged across participants, are shown in Figure 3 (preference in panel A, typicality in panel B). We obtained $95 \%$ confidence intervals about each point in the time series using a bootstrapping procedure (Efron \& Tibshirani, 1994). A comparison of the plots by preexposure and task indicated a very similar pattern across conditions, with all plots showing an increasing tendency for the eye to be directed toward the chosen item prior to the response. To further compare the rate of increase in gaze bias across conditions, linear fits were performed for each bootstrap sample in order to compute the average and confidence intervals of the slope in each condition. Most importantly, there was no support for the gaze cascade model's critical prediction that for preference decisions there would be a steeper rate of increase in gaze bias for items that were preexposed than for items that were not (both slopes $=$ 0.16 , n.s.).

As in Glaholt and Reingold (in press), we supplemented the gaze likelihood analysis with a fine-grained dwell sequence analysis. For the purpose of this analysis, a dwell is defined as the cumulative duration of all con- 


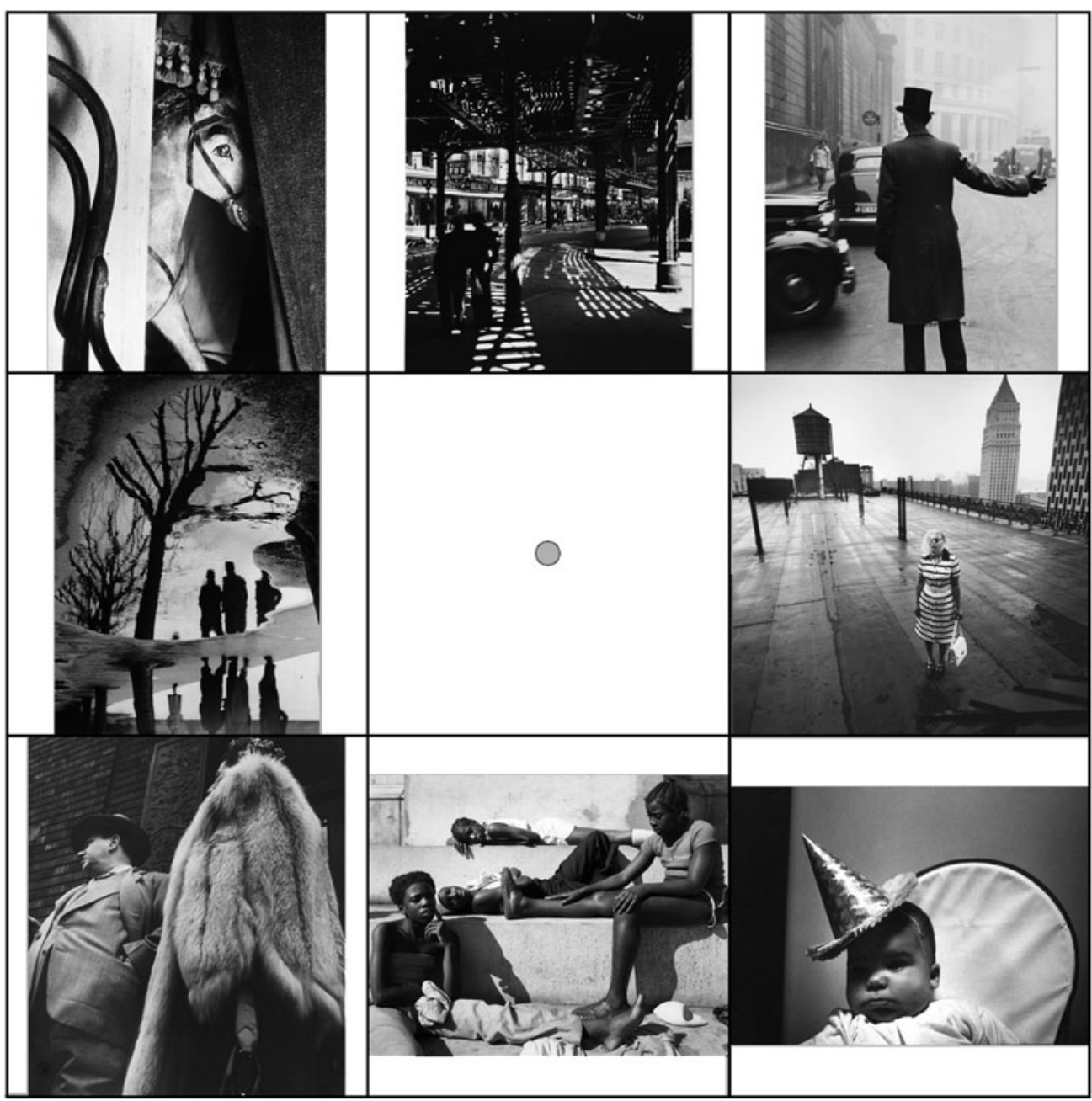

Figure 2. Stimulus display for the 8-AFC task. Prior to each 8-AFC display, four of the eight images were shown to the participant, one after another. When the 8-AFC display was presented, participants chose the photograph they preferred (preference) or the photograph that was the most unusual (typicality).

secutive fixations from the moment the participant's gaze enters a grid square containing an image, and until it exits that square. The dwell duration measure is similar to the measure of gaze duration used in reading research, which corresponds to the cumulative duration of consecutive fixations on a word during the initial processing (i.e., first pass) of that word (see Rayner, 1998). However, unlike gaze duration, the term $d$ well does not distinguish between initial and subsequent visits on an item.

As shown in Figure 3, we created four different dwell position bins going back from the response: the last two dwell positions prior to response (last), Positions 3-4 prior to the response $(-1)$, Positions $5-6$ prior to response $(-2)$, and Positions $7-8$ prior to response $(-3)$. In addition, in order to examine early gaze bias effects, we created a dwell position bin corresponding to the first two dwell positions in the trial (first). For each bin, we computed the mean dwell duration for dwells on the chosen item and dwells on the other items, and we also computed the proportion of dwells directed toward the chosen item.
These measures were computed separately for items that were preexposed and those that were not, as well as for each decision task (see Figure 3, panels C, D, E, and F).

In analyzing dwell duration, we conducted a $2 \times 2 \times$ $2 \times 5$ mixed ANOVA that crossed decision (preference, typicality) as a between-participants variable, and choice (chosen, other), preexposure (preexposed, not preexposed), and dwell position bin (first, $-3,-2,-1$, last) as within-participants variables (see Figure 3, panels C and D). Replicating the bias in dwell duration reported by Glaholt and Reingold (in press), dwells on the chosen item were longer than dwells on other items $[F(1,30)=$ $\left.108.13, M S_{\mathrm{e}}=2.19 \times 10^{5}, p<.001\right]$. Planned comparisons showed that this gaze bias was significant in each dwell position bin (all $F_{\mathrm{S}}>34.15$, all $p \mathrm{~s}<.001$ ). Dwells on items not preexposed were significantly longer than dwells on preexposed items $\left[F(1,30)=34.21, M S_{\mathrm{e}}=\right.$ $\left.1.11 \times 10^{5}, p<.001\right]$, an effect in the direction opposite that predicted by the gaze cascade model. Planned comparisons indicated that the effect of preexposure was 
A

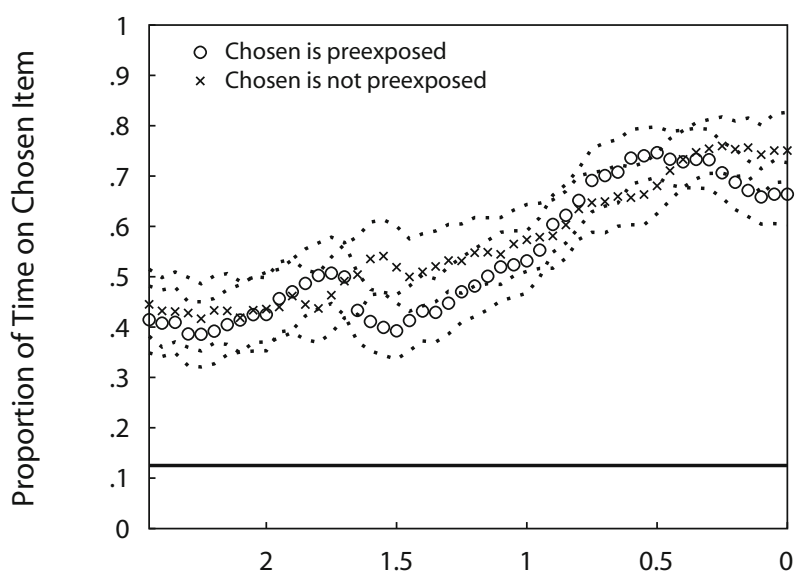

Time Until Response (sec)

\section{C}

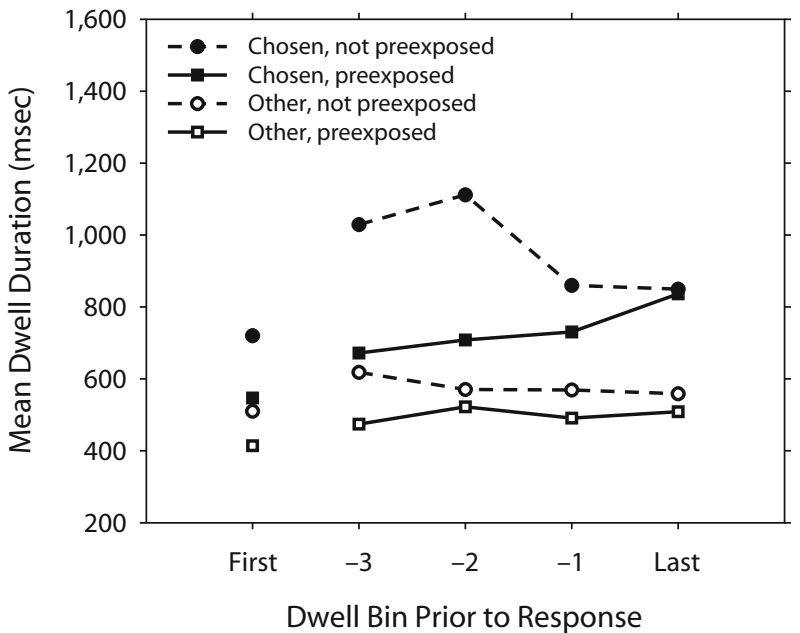

E

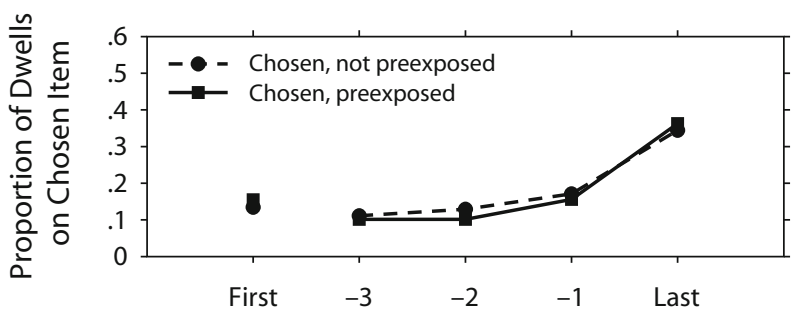

Dwell Bin Prior to Response
B

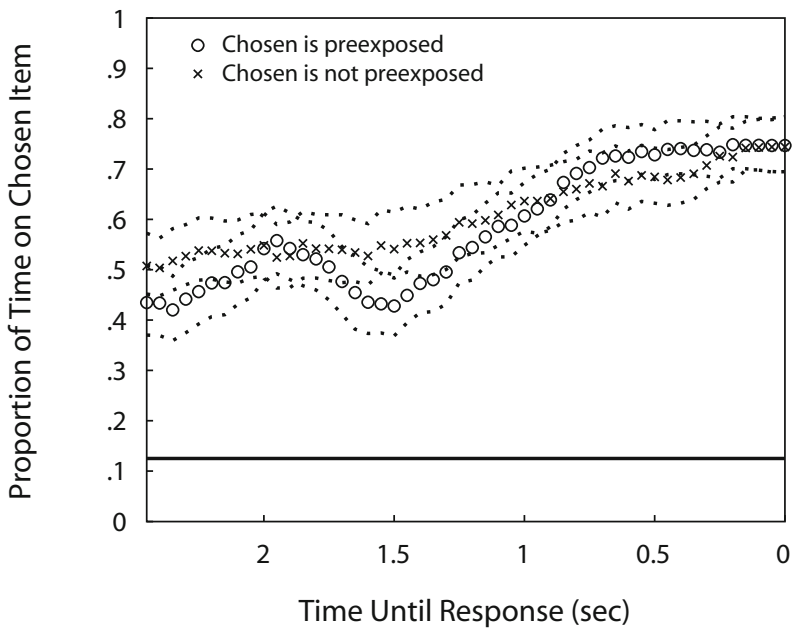

D

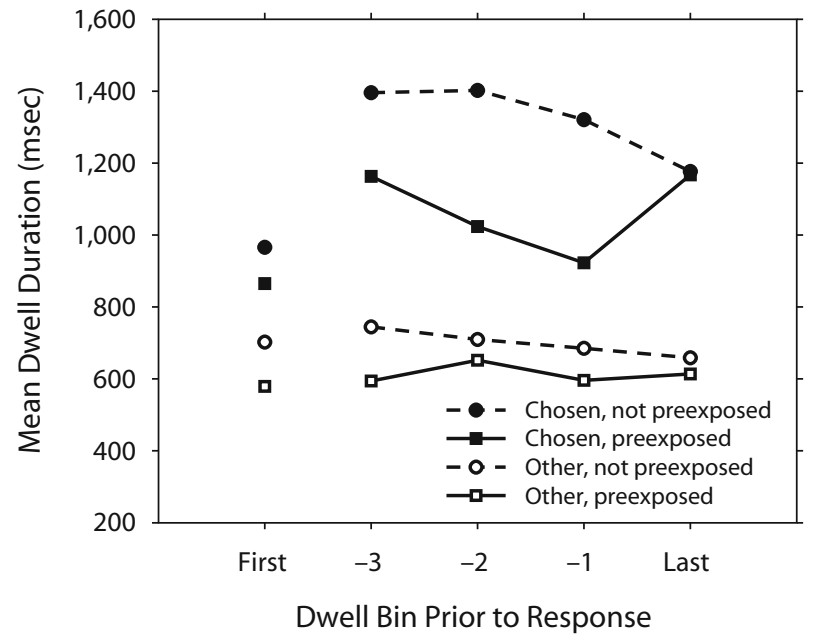

$\mathbf{F}$

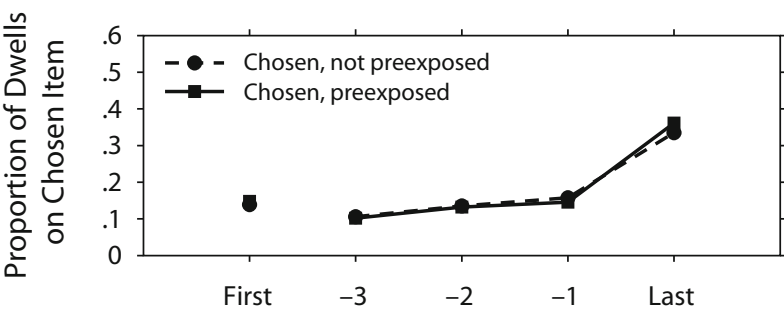

Dwell Bin Prior to Response

Figure 3. Analyses of gaze bias: (1) Gaze likelihood analysis, plotting the proportion of time that gaze was directed towards the chosen item, going back $2.5 \mathrm{sec}$ from the response, as a function of preexposure (preference in panel A, typicality in panel B). The solid line represents the proportion of time that gaze would be directed to the chosen item by chance (.125); (2) Mean dwell duration for the chosen and other items, as a function of preexposure (PE), for each of 4 dwell bins prior to the response, and for the first dwell bin (preference in panel C, typicality in panel D); (3) Proportion of dwells directed towards the chosen item, as a function of preexposure (PE), for each of 4 bins prior to the response, and for the first dwell bin (preference in panel $\mathbf{E}$, typicality in panel $\mathrm{F}$ ).

significant in all but the final bin, where the preexposure effect was eliminated (first, $-3,-2,-1$, all $F \mathrm{~s}>5.45$, all $p \mathrm{~s}<.05$; last, $F=1.08, p=.31$ ). Most importantly, in marked contrast to the prediction of the gaze cascade model that gaze bias should be stronger for preexposed items, we found a larger bias in dwell duration for items 
not preexposed $\left[F(1,30)=6.12, M S_{\mathrm{e}}=1.13 \times 10^{5}\right.$, $p<.05]$.

Dwells were longer overall for typicality decisions than for preference decisions $\left[F(1,30)=4.79, M S_{\mathrm{e}}=1.79 \times\right.$ $\left.10^{6}, p<.05\right]$, and the bias in dwell duration toward the chosen item was larger for the typicality decision than for the preference decision $\left[F(1,30)=7.60, M S_{\mathrm{e}}=2.19 \times\right.$ $\left.10^{5}, p<.05\right]$, a finding that also contradicts the gaze cascade model, which predicts that the gaze bias should be larger in preference decisions than in other kinds of decisions. No other interactions with decision type approached significance (all $F \mathrm{~s}<1)$.

To analyze dwell frequency toward the chosen item, we conducted a $2 \times 2 \times 5$ mixed ANOVA that crossed decision (preference, typicality) as a between-participants variable, and preexposure (preexposed, not preexposed) and dwell position bin (first, $-3,-2,-1$, last) as withinparticipants variables (see Figure 3, panels E and F). The findings replicated the pattern reported by Glaholt and Reingold (in press), where toward the end of the trial dwell frequency was substantially biased toward the chosen item $\left[F(4,120)=123.02, M S_{\mathrm{e}}=0.005, p<.001\right]$. Planned comparisons indicated that dwell frequency in the last two bins was significantly greater than chance (collapsed across task and preexposure, both $t \mathrm{~s}>3.18, p \mathrm{~s}<.01$ ). Contrary to the prediction of the gaze cascade model, the bias in dwell frequency was not affected either by decision or by preexposure (both $F \mathrm{~s}<1$ ).

\section{DISCUSSION}

We tested several predictions derived from the gaze cascade model of preference decision making (Shimojo et al., 2003; Simion \& Shimojo, 2006, 2007). The model postulated a feedback loop between mere exposure and preferential looking, whereby exposure to a stimulus increases preference for that stimulus, which in turn increases the likelihood of looking at that stimulus. In our experiment we preexposed half of the alternatives prior to an 8-AFC decision. On the basis of the gaze cascade model, preexposed stimuli were expected to be chosen more often. Preexposure was also predicted to produce stronger biases in looking behavior. In addition, the model predicts this pattern of biases to be substantially smaller, or absent in nonpreference decisions. In contrast to these predictions, we found that dwells were not directed to preexposed items more often than to items not preexposed, and dwells on the preexposed items were actually shorter in duration than those on items not preexposed. Most importantly, preexposed items were not more likely to be chosen than items that were not preexposed, and the magnitude of the bias in dwell duration was actually larger for items that were not preexposed.

In addition, in both the present study and in our previous work (Glaholt \& Reingold, in press) we demonstrated remarkably similar patterns of gaze biases across the preference decisions and the recency and typicality control tasks. This is in marked contrast to the preference specificity predicted by the gaze cascade model and the findings of differences between the preference task and the roundness control task reported by Shimojo and colleagues (Shimojo et al., 2003; Simion \& Shimojo, 2006). Differences in the level of encoding across tasks might underlie this discrepancy. Specifically, preference, recency, and typicality might involve deeper encoding of semantic features, whereas the roundness task might primarily entail surface-level encoding. Regardless of this speculation, the absence of task dissociations in our studies is clearly inconsistent with the preference specificity predicted by the gaze cascade model. We therefore argue that the gaze biases, as demonstrated both by Shimojo and colleagues and in our studies, reflect a general characteristic of visual decision making rather than a preference-specific mechanism, as proposed by the gaze cascade model.

It is important to note that our failure to find the predicted effects of preexposure is not likely to be due to a lack of strength in our manipulation of preexposure. This is because the preexposure manipulation produced strong effects in our study, although not the ones predicted by the gaze cascade model. Future studies employing other types of selective exposure of items and/or manipulations of preexisting preferences are required to further test the gaze cascade model. Regardless of the results of such future experiments, it is clear that the current formulation of the gaze cascade model is insufficient to account for our findings.

Several aspects of our results merit further investigation. We found that the bias in dwell duration toward the chosen item was larger for items not preexposed than for preexposed ones. One possible explanation for this is that the bias in dwell duration reflects a process of selective encoding of stimuli in terms of their relevance to the decision task. Specifically, the processing of relevant rather than less relevant stimulus alternatives might entail greater perceptual (e.g., stimulus encoding) and postperceptual (e.g., evaluation, memory) load, resulting in longer dwell durations on the former than on the latter. It is possible that in the present study, preexposed stimuli were partly encoded with respect to task relevance prior to the 8-AFC decision, whereas the stimuli not preexposed could have been differentiated only during the 8-AFC decision.

Regardless of the explanation for the dwell duration bias, it is clear that, whereas dwell duration was sensitive to stimulus preexposure, frequency of visiting the chosen item was not. This dissociation suggests that the bias in dwell duration and the dwell frequency bias might index independent aspects of visual decision making. This dissociation provides support for frameworks of decision making which postulate multiple stages, processes, or strategies (e.g., Payne, 1976; Payne, Bettman, Coupey, \& Johnson, 1992; Russo \& Leclerc, 1994; Senter \& Wedell, 1999; Wedell \& Senter, 1997; see Ford, Schmitt, Schechtman, Hults, \& Doherty, 1989, for a review). Our finding of gaze bias in dwell duration but not in dwell frequency early in the trial might reflect the operation of an early screening stage, where items are quickly encoded and the set of potentially relevant alternatives is reduced. In 
contrast, the bias in dwell frequency might reflect the operation of a later evaluative stage, involving active comparison across alternatives and resulting in higher dwell frequency on the chosen item.

Thus, although the present study failed to provide support for the specific model proposed by Shimojo and colleagues, it clearly demonstrated the potential usefulness of the methodology they introduced for the broader investigation of the various processes and stages that might underlie visual decision making.

\section{AUTHOR NOTE}

We thank Jiye Shen for essential technical assistance in these experiments. This research was funded by an NSERC research grant to E.M.R. Please address all correspondence to M. G. Glaholt or E. M. Reingold, University of Toronto at Mississauga, Department of Psychology, 3359 Mississauga Road N., RM 2037B, Mississauga, ON, L5L 1C6Canada (e-mail: mackenzie.glaholt@gmail.com or reingold@psych utoronto.ca).

\section{REFERENCES}

Birch, E. E., Shimojo, S., \& Held, R. (1985). Preferential looking assessment of fusion and stereopsis in infants aged 1 to 6 months. Investigative Ophthalmology \& Visual Science, 26, 366-370.

Efron, B., \& Tibshirani, R. J. (1994). An introduction to the bootstrap. Boca Raton, FL: Chapman \& Hall.

FANTZ, R. L. (1964). Visual experience in infants: Decreased attention to familiar patterns relative to novel ones. Science, 146, 668-670. doi:10.1126/science.146.3644.668

Ford, J. K., Schmitt, N., Schechtman, S. L., Hults, B. M., \& DoHerty, M. L. (1989). Process tracing methods: Contributions, problems, and neglected research questions. Organizational Behavior \& Human Decision Processes, 43, 75-117. doi:10.1016/0749 -5978(89)90059-9

Glaholt, M. G., \& Reingold, E. M. (in press). The time course of gaze bias in visual decision tasks. Visual Cognition.

Kunst-Wilson, W. R., \& Zajonc, R. B. (1980). Affective discrimination of stimuli that cannot be recognized. Science, 207, 557-558. doi: $10.1126 /$ science. 7352271
Moreland, R. L., \& ZaJonc, R. B. (1977). Is stimulus recognition a necessary condition for the occurrence of exposure effects? Journal of Personality \& Social Psychology, 35, 191-199. doi:10.1037/0022 $-3514.35 .4 .191$

Moreland, R. L., \& Zajonc, R. B. (1982). Exposure effects in person perception: Familiarity, similarity, and attraction. Journal of Experimental Social Psychology, 18, 395-415. doi:10.1016/0022 -1031(82)90062-2

PAYNe, J. W. (1976). Task complexity and contingent processing in decision making: An information search and protocol analysis. Organizational Behavior \& Human Performance, 16, 366-387. doi:10.1016/0030-5073(76)90022-2

Payne, J. W., Bettman, J. R., Coupey, E., \& Johnson, E. J. (1992). A constructive process view of decision making: Multiple strategies in judgment and choice. Acta Psychologica, 80, 107-141. doi:10.1016/0001-6918(92)90043-D

RAYNER, K. (1998). Eye movements in reading and information processing: 20 years of research. Psychological Bulletin, 102, 21-38. doi:10.1037/0033-2909.124.3.372

Russo, J. E., \& LECLERC, F. (1994). An eye-fixation analysis of choice processes for consumer nondurables. Journal of Consumer Research, 21, 274-290. doi:10.1086/209397

Senter, S. M., \& Wedell, D. H. (1999). Information presentation constraints and the adaptive decision maker hypothesis. Journal of Experimental Psychology: Learning, Memory, \& Cognition, 25, 428446. doi:10.1037/0278-7393.25.2.428

Shimojo, S., Simion, C., Shimojo, E., \& Scheier, C. (2003). Gaze bias both reflects and influences preference. Nature Neuroscience, $\mathbf{6}$, 1317-1322. doi:10.1038/nn1150

Simion, C., \& Shimojo, S. (2006). Early interactions between orienting, visual sampling and decision making in facial preference. Vision Research, 46, 3331-3335. doi:10.1016/j.visres.2006.04.019

Simion, C., \& Shimojo, S. (2007). Interrupting the cascade: Orienting contributes to decision making even in the absence of visual stimulation. Perception \& Psychophysics, 69, 591-595.

Wedell, D. H., \& Senter, S. M. (1997). Looking and weighting in judgment and choice. Organizational Behavior \& Human Decision Processes, 70, 41-64. doi:10.1006/obhd.1997.2692

ZAJONC, R. B. (1968). Attitudinal effects of mere exposure. Journal of Personality \& Social Psychology, 9, 1-27. doi:10.1037/h0025848

(Manuscript received August 15, 2008; revision accepted for publication October 4, 2008.) 